\title{
REMARKS ON A COMPARISON THEOREM FOR SCALAR RICCATI EQUATIONS
}

\author{
C. C. TRAVIS
}

ABSTRACT. A comparison theorem for scalar Riccati equations will be stated which contains a recent theorem of Stafford and Heidel.

In a recent paper [1], Stafford and Heidel obtain a comparison theorem for the scalar Riccati equations

$$
\begin{aligned}
& r^{\prime}(t)+r^{2}(t)+q_{1}(t)=0, \\
& s^{\prime}(t)+s^{2}(t)+q_{2}(t)=0 .
\end{aligned}
$$

Theorem (Stafford and Heidel). Suppose that $q_{1}$ and $q_{2}$ are nonnegative on $[a, b)$ where $0<a<b \leq \infty$ and that

$$
\int_{a}^{t} \tau^{2} q_{2}(\tau) d \tau \leq \int_{a}^{t} \tau^{2} q_{1}(\tau) d \tau, \quad t \geq a .
$$

If (1) has a solution $r(t)$ on $[a, b)$ such that $a r(a)<1$ then (2) has a solution on $[a, b)$.

The following theorem is an improvement over the result of Stafford and Heidel.

Theorem 1. Suppose that (1) has a solution $r(t)$ on $[a, b)$ where $0<a<$ $b \leq \infty$, and that there exists a constant $s_{0}$ such that

$$
\left|d\left(1-a s_{0}\right)+\int_{a}^{t} \tau^{2} q_{2}(\tau) d \tau\right| \leq d(1-a r(a))+\int_{a}^{t} \tau^{2} q_{1}(\tau) d \tau .
$$

Then (2) has a solution $s(t)$ on $[a, b)$ such that $s(a)=s_{0}$ and $|1-t s(t)| \leq 1$ $-\operatorname{tr}(t)$.

Proof. The outline of the proof that we present here follows Hille [2, p. 462]. Consider the integral equations

$$
\begin{gathered}
t z(t)=a z(a)+\int_{a}^{t} z^{2}(\tau) d \tau+\int_{a}^{t} \tau^{2} q_{1}(\tau) d \tau \\
t v(t)=a\left(1-a s_{0}\right)+\int_{a}^{t} v^{2}(\tau) d \tau+\int_{a}^{t} \tau^{2} q_{2}(\tau) d \tau .
\end{gathered}
$$

Received by the editors February 25, 1974 and, in revised form, July 3, 1974. AMS (MOS) subject classifications (1970). Primary 34A15, 34C10.

Key words and phrases. Riccati equation, comparison theorem, disconjugacy. 
It is easily verified that (4) has the solution $z(t)=1-\operatorname{tr}(t)$ on $[a, b)$ which is the limit of a strictly increasing sequence $\left\{z_{n}(t)\right\}$ of successive approximations satisfying

$$
\begin{aligned}
& t z_{n}(t)=a z(a)+\int_{a}^{t} z_{n-1}^{2}(\tau) d \tau+\int_{a}^{t} \tau^{2} q_{1}(\tau) d \tau, \\
& z_{0}(t)=a z(a)+\int_{a}^{t} \tau^{2} q_{1}(\tau) d \tau .
\end{aligned}
$$

If we establish that (5) has a solution $v(t)$ existing on $[a, b)$, then $s(t)=$ $(1-v(t)) / t$ is a solution of (2) existing on $[a, b)$ satisfying $s(a)=s_{0}$. A solution of $(5)$ can be constructed as the limit of the sequence $\left\{v_{n}(t)\right\}$ of solutions to

$$
\begin{aligned}
& t v_{n}(t)=a\left(1-a s_{0}\right)+\int_{a}^{t} v_{n-1}^{2}(\tau) d \tau+\int_{a}^{t} \tau^{2} q_{2}(\tau) d \tau, \\
& v_{0}(t)=a\left(1-a s_{0}\right)+\int_{a}^{t} \tau^{2} q_{2}(\tau) d \tau .
\end{aligned}
$$

The convergence of the sequence $\left\{v_{n}(t)\right\}$ follows from the inequalities

$$
\left|v_{n}(t)\right| \leq z_{n}(t), \quad\left|v_{n+1}(t)-v_{n}(t)\right| \leq z_{n+1}(t)-z_{n}(t)
$$

Corollary 1. If (1) has a solution $r(t)$ on $[a, b)$ such that $a r(a) \leq 1$ and

$$
\left|\int_{a}^{t} \tau^{2} q_{2}(\tau) d \tau\right| \leq \int_{a}^{t} \tau^{2} q_{1}(\tau) d \tau, \quad t \geq a,
$$

then (2) has a solution $s(t)$ on $[a, b)$ such that $0<1-t s(t) \leq 1-\operatorname{tr}(t)$.

Proof. Choose $s_{0}$ such that $a r(a) \leq a s_{0} \leq 1$. Condition (3) now follows from (6) and the fact that $0 \leq 1-a s_{0} \leq 1-\operatorname{ar}(a)$.

In order to obtain a result which complements Theorem 2 of [1], define $z(t)=\mu^{\prime}(t)-\mu(t) r(t)$ and $v(t)=\mu^{\prime}(t)-\mu(t)_{s}(t)$ where $\mu(t)$ is a positive function of class $C^{2}[a, b)$. Then $z(t)$ and $v(t)$ satisfy

$$
\begin{aligned}
& \mu(t) z(t)=\mu(a) z(a)+\int_{a}^{t} \mu^{\prime \prime}(\tau) \mu(\tau) d \tau+\int_{a}^{t} z^{2}(\tau) d \tau+\int_{a}^{t} \mu^{2}(\tau) q_{1}(\tau) d \tau \\
& \mu(t) v(t)=\mu(a) v(a)+\int_{a}^{t} \mu^{\prime \prime}(\tau) \mu(\tau) d \tau+\int_{a}^{t} v^{2}(\tau) d \tau+\int_{a}^{t} \mu^{2}(\tau) q_{2}(\tau) d \tau
\end{aligned}
$$

The following theorem can now be proven:

Theorem 2. Suppose that (1) has a solution $r(t)$ on $[a, b)$ where $0<a<$ $b \leq \infty$, and that there exists a constant $s_{0}$ such that

$$
\left|\mu(a)\left(\mu^{\prime}(a)-\mu(a) s_{0}\right)+\int_{a}^{t} \mu^{2}(\tau) q_{2}(\tau) d \tau\right|
$$

$$
\leq \mu(a)\left(\mu^{\prime}(a)-\mu(a) r(a)\right)+\int_{a}^{t} \mu^{2}(\tau) q_{1}(\tau) d \tau, \quad t \geq a_{3}
$$


where $\mu(t)$ is a positive function of class $C^{2}[a, b)$. Then (2) has a solution $s(t)$ on $[a, b)$ such that $s(a)=s_{0}$ and $\left|\mu^{\prime}(t)-\mu(t) s(t)\right| \leq \mu^{\prime}(t)-\mu(t) r(t)$. If in addition, we assume that $\mu(a) r(a) \leq \mu^{\prime}(a)$, then condition (9) can be replaced by

$$
\left|\int_{a}^{t} \mu^{2}(\tau) q_{2}(\tau) d \tau\right| \leq \int_{a}^{t} \mu^{2}(\tau) q_{1}(\tau) d \tau .
$$

Theorem 2 can be used to obtain several new oscillation and disconjugacy criteria for the equation

$$
Y^{\prime \prime}(t)+q_{1}(t) Y(t)=0
$$

for example:

$\frac{1}{4}<\frac{1}{t-a} \int_{a}^{t} \tau^{2} q_{1}(\tau) d \tau \rightarrow$ oscillation, $\left|\frac{1}{t-a} \int_{a}^{t} \tau^{2} q_{1}(\tau) d \tau\right|<\frac{1}{4} \rightarrow$ disconjugacy.

Theorem 2 can also be used to compare the disconjugacy of (11) with that of

$$
Y^{\prime \prime}(t)+q_{2}(t) Y(t)=0
$$

Theorem 3. If equation (11) is disconjugate on $[a, \infty)$ and

$$
\left|\int_{a}^{t}(\tau-a)^{a} q_{2}(\tau) d \tau\right| \leq \int_{a}^{t}(\tau-a)^{\alpha} q_{1}(\tau) d \tau, \quad t \geq a .
$$

holds for some $\alpha \geq 2$, then equation (12) is disconjugate on $[a, \infty)$.

Proof. Let $Y(t)$ be the solution of (11) satisfying $Y(a)=0, Y^{\prime}(a)=1$. Since equation (11) is disconjugate $Y(t)>0$ for $t>a$ and $r(t)=Y^{\prime}(t) / Y(t)$ is a solution of (1) existing on $(a, \infty)$. Condition (13) implies that $q_{1}(t) \geq 0$ in a positive neighborhood of $a$, from which it follows that

$$
\lim _{t \rightarrow a^{+}} \sup _{t}(t-a) r(t) \leq 1
$$

(see the proof of Lemma 9.4.1 in [2, p. 457]). Now define $\mu(t)=(t-a)^{\alpha / 2}$ and verify that for $a \geq 2$ we have $\mu(a)_{r}(a) \leq \mu^{\prime}(a)$. The result now follows from Theorem 2.

In Example 3 of [1], Stafford and Heidel obtained the following sufficient condition for the disconjugacy of $(11)$ on $[0, \infty)$ :

$$
-\frac{3}{4} \leq \frac{1}{t} \int_{0}^{t} \tau^{2} q_{1}(\tau) d \tau \leq \frac{1}{4}
$$

We will obtain a generalization of this condition with the aid of some observations made by $A$. Wintner [3] and the following lemma which we present without proof.

Lemma 1. Equation (11) is disconjugate on $[0, \infty)$ if and only if there exists a function $w(t)$ of class $C^{1}[0, \infty)$ satisfying the inequality 


$$
(t w(t))^{\prime} \geq w^{2}(t)+t^{2} q_{1}(t) \quad \text { for } t \in[0, \infty)
$$

Theorem 4. Suppose that there exists a nonnegative function $b(t)$ such that

$$
\left|\int_{0}^{t} \tau^{2}\left[q_{1}(\tau)+b(\tau)\right] d \tau\right| \leq t^{2} b^{1 / 2}(t) \quad \text { for } t \in[0, \infty)
$$

then equation (11) is disconjugate on $[0, \infty)$.

Proof. Assuming the condition of the theorem, a direct substitution shows that $(15)$ is satisfied by

$$
w(t)=\frac{1}{t} \int_{0}^{t} \tau^{2} q_{1}(\tau) d \tau+\frac{1}{t} \int_{0}^{t} \tau^{2} b(\tau) d \tau .
$$

Notice that condition (14) now follows from Theorem 4 with the choice $b(t)=$ $1 / 4 t^{2}$.

Acknowledgement. I would like to thank Professor Zeev Nehari for some helpful discussions concerning Theorem 3.

\section{REFERENCES}

1. Stafford and Heidel, A new comparison theorem for scalar Riccati equations, Bull. Amer. Math. Soc. 80 (1974), 754-757.

2. E. Hille, Lectures on ordinary differential equations, Addison-Wesley, Reading, Mass., 1969. MR $40 \# 2939$.

3. A. Wintner, On the non-existence of conjugate points, Amer. J. Math. 73 (1951), 368-380. MR 13, 37 .

DEPARTMENT OF MATHEMATICS, UNIVERSITY OF TENNESSEE, KNOXVILLE, TENNESSEE 37916 